\title{
Études/Inuit/Studies
}

\section{La contribution de Patrick Plumet à l'archéologie québécoise}

\section{Gilles Tassé}

Volume 39, numéro 2, 2015

URI : https://id.erudit.org/iderudit/1038154ar

DOI : https://doi.org/10.7202/1038154ar

Aller au sommaire du numéro

Éditeur(s)

Centre interuniversitaire d'études et de recherches autochtones (CIÉRA)

ISSN

0701-1008 (imprimé)

1708-5268 (numérique)

Découvrir la revue

Citer ce document

Tassé, G. (2015). La contribution de Patrick Plumet à l'archéologie québécoise. Études/Inuit/Studies, 39(2), 307-308. https://doi.org/10.7202/1038154ar d'utilisation que vous pouvez consulter en ligne.

https://apropos.erudit.org/fr/usagers/politique-dutilisation/ 


\section{La contribution de Patrick Plumet à l'archéologie québécoise}

Qu'on me permette d'abord de mentionner les aspects de ma collaboration avec Patrick Plumet qui m'ont touché de façon intime. J'enseignais au Collège Sainte-Marie quand celui-ci est devenu d'office partie du réseau de l'Université du Québec, créé en 1969. J'avais à ce moment terminé la scolarité d'un doctorat en archéologie préhistorique à la Sorbonne, avec l'art rupestre comme sujet de thèse. On m'a donc rattaché à la section d'histoire de l'art de l'Université du Québec à Montréal (UQÀM).

Certains collègues ont entamé à ce moment des intrigues visant à remplacer deux membres de cette section par des copains. Patrick a réagi avec vigueur contre ce genre de procédés. Finalement, un des intéressés a réagi en trouvant un poste à l'Université de Montréal, tandis que notre directeur de département, qui penchait dans le même sens que Patrick, me faisait discrètement signer une demande de congé pour terminer ma thèse de doctorat. Entre-temps, Patrick avait créé le Laboratoire d'archéologie, qui fut l'occasion d'une fructueuse et loyale collaboration entre nous, et un milieu propice aux recherches, attirant chargés de cours et étudiants. Son rattachement au département de Géologie et des Sciences de l'atmosphère rétablit une tranquillité propice au travail.

C'est alors que Claire Mousseau, du ministère des Affaires culturelles du Québec, sollicita l'aide du Laboratoire d'archéologie de l'UQÀM pour organiser une école de fouilles à Grandes-Bergeronnes, sur la Haute-Côte-Nord, où plusieurs sites préhistoriques amérindiens étaient menacés par l'érosion. Grâce à l'entregent de Patrick et à ses talents d'organisateur, l'École de fouilles de l'UQAM devint un lieu de collaboration entre disciplines: archéologie, géologie, palynologie, datations, vestiges végétaux, restes osseux, etc. Des collègues de l'Université de Montréal, Normand Clermont et Claude Chapdelaine, apportèrent leur expérience des sites amérindiens, en particulier celui de Pointe-du-Buisson, dans l'analyse des vestiges céramiques de la région des Bergeronnes. Nos collègues des Sciences de la terre, Gilbert Prichonnet et Robert-André Daigneault, assumèrent l'établissement de la carte géologique de la région, tandis qu'Hélène Gauvin et Marie-France Archambault dirigeaient des fouilles complétant celles de Grandes-Bergeronnes à Tadoussac et à Baie Sainte-Catherine. Marie-France Archambault avait déjà réalisé une évaluation du site de la Falaise, entamé par l'érosion, dont je devais, avec l'aide de Serge Lebel pour la partie ouest, m'occuper après le lancement des travaux du site Lavoie, que Patrick puis JeanFrançois Moreau, ont dirigé. Je dois aussi mentionner la participation de deux spécialistes en palynologie, Pierre Richard et Pierre Gangloff, du département de Géographie de l'Université de Montréal, ainsi que celle d'Alayn Larouche, pour l'analyse des restes végétaux. L'Ostéothèque de Montréal, logée à l'époque dans les locaux de notre Laboratoire d'archéologie, réalisa l'analyse des vestiges osseux animaux sous la direction de Michèle Courtemanche. 
Ces recherches et cette collaboration entre institutions ${ }^{1}$ se sont déroulées de façon harmonieuse grâce au talent de Patrick Plumet pour l'organisation des rapports humains où les compétences de chaque spécialiste étaient à la fois reconnues et utilisées au mieux. Patrick a également lancé, en marge de la création du Laboratoire d'archéologie, la publication de Paléo-Québec, qui traitait aussi bien d'archéologie arctique que de la préhistoire du Québec en général. On y retrouve comme auteurs plusieurs universitaires québécois.

En guise de conclusion, on pourrait ajouter que le dynamisme et l'apport scientifique de Patrick Plumet ont honoré l'archéologie québécoise, et que ce fut pour moi un honneur de compter parmi ses collaborateurs.

Gilles Tassé

\section{Chemins d'étoiles}

Heureux l'homme qui rencontre Louis-Edmond Hamelin dans son jeune âge, pas besoin de radar, il se dirigera vers le nord. Patrick a été de ceux-là. Patrick était un être fragile et fort, vif et nerveux comme un oiseau que la neige inquiète, mais voilà que sa direction est trouvée. C'était après la guerre de 1939-1945, il immigrait au Québec.

En 1969, l'Université du Québec à Montréal (UQÀM) est créée. En 1973, Patrick Plumet y est pour enseigner l'archéologie et moi, Yolande Simard, 45 ans, j'y suis aussi. C'était l'œuvre de la «Révolution Tranquille». Qui est cette femme? Que fait-elle en archéologie? Une intruse? Une agente de la GRC (Gendermerie Royale du Canada)? Ou de l'université? La guerre n'était pas loin pour Patrick qui l'avait vécue en Afrique du Nord. Sa curiosité de chercheur le pousse à me le demander abruptement. Je lui réponds que je suis une femme de la Terre et que l'homme sur la Terre et en dessous m'intéresse. Pour appuyer ma réponse, je l'invite à venir chez moi pour y rencontrer mon époux et mes deux enfants. Par la suite, je ne semblais plus être une espionne... je pouvais donc avoir accès au code Borden. Par-delà les ans, nos contacts se sont répétés dans une harmonie normale et sereine.

Patrick, comme ceux qu'au XVII ${ }^{\mathrm{e}}$ siècle en Nouvelle-France on appelait «les voyageurs», ira découvrir et décoder les particularités techniques et culturelles des peuples sans écriture du Grand Nord dans la zone arctique du temps et de l'espace des préhistoriques. Ayant fouillé un site présumé vieux de plus de deux mille ans en Arctique de l'Est, il est toujours resté coi devant la question du film D'où sont venus les Dorsétiens?, alors que moi, sans réputation à défendre, j'osais dire qu'avec mes recherches par tâtonnements, il m'apparaissait possible que le haut crâne caréné des

1 Soit l'Université du Québec à Montréal et l'Université du Québec à Chicoutimi — après le passage de Jean-François Moreau à cette dernière —, l'Université de Montréal, le ministère des Affaires culturelles, auxquels il faut ajouter les dirigeants locaux, comme le maire de Grandes-Bergeronnes, Gaétan Boucher. 